\title{
41. E.E.G. Abnormarities in Hyponatremia, appeared after Pituitary Stalk Section
}

\author{
Sakae Fukuda and Kensuke KuroKaWA \\ Department of Neurosurgery, Shizuoka Red Cross Hospital \\ Takashi Mimura \\ Department of Surgery, Shizuoka Red Cross Hospital \\ Nobuo YosHII \\ Department of Neurosurgery, Keio University
}

48 year-old, female, with recurrent breast cancer, was performed radica mastectomy 13 years ago.

In March, 1967, pituitary stalk section was performed on the patient for th treatment of the recurrent breast cancer.

On 7 th and 18th post operative day, loss of consciousness was appeared du to hyponatremia.

EEG tracings taken during second attack of unconsciousness, showed diffus $\delta$ and $\theta$ waves in all areas with occasional $\beta$ waves, particularly in frontal area. In both unipolar and bipolar leads, there disclosed slow waves of 30 to $80 \mu \mathrm{V}$ in lef temporal area and of 15 to $30 \mu \mathrm{V}$ in right temporal area. All these tracings re vealed the fact that right side is slower in frequency.

This unconsciousness disappeared rapidly after large amount of I-V salin solution. In following EEG taken on 7 th, 20th, and 180th day after second attack there still noticed slow waves, although diminished in frequency gradually.

After 6 months, all EEG became within normal limits.

The slow wave focus in right frontal and temporal area were found through al tracings, but this may be due to the surgery.

\section{Experimental Studies of Cerebral Hemodynamics during Intracranial Hypertention}

\author{
Hajime NagaI, Masahiro Furuse, Muneyoshi KaYo, Yoshihiko OKa, \\ Mitsuo Sugiura, Atsushi Ikeyama, Shigeru Maeda, \\ Michiaki Hasuo and Shin Hoshikawa \\ 2nd Department of Surgery, School of Medicine, Nagoya University
}

The relationship between intracranial hypertension and cerebral hemodynamis was investigated. Respiration, systemic blood pressure, cerebral blood flow by he: 